\title{
miR-205 regulates basement membrane deposition in human prostate: implications for cancer development
}

\author{
P Gandellini ${ }^{1}$, V Profumo ${ }^{1}$, A Casamichele ${ }^{1}, \mathrm{~N}$ Fenderico ${ }^{1}, \mathrm{~S}$ Borrelli ${ }^{2}$, G Petrovich ${ }^{2}$, G Santilli ${ }^{1}$, M Callari $^{1}$, M Colecchia $^{3}$, S Pozzi $^{2}$, \\ M De Cesare ${ }^{1}$, M Folini ${ }^{1}$, R Valdagni ${ }^{4,5}$, R Mantovani $^{2}$ and N Zaffaroni ${ }^{\star, 1}$
}

The basement membrane (BM) is a layer of specialized extracellular matrix that surrounds normal prostate glands and preserves tissue integrity. Lack or discontinuity of the BM is a prerequisite for tumor cell invasion into interstitial spaces, thus favoring metastasis. Therefore, BM maintenance represents a barrier against cancer development and progression. In the study, we show that miR-205 participates in a network involving $\Delta N p 63 \alpha$, which is essential for maintenance of the BM in prostate epithelium. At the molecular level, $\Delta N p 63 \alpha$ is able to enhance miR-205 transcription by binding to its promoter, whereas the microRNA can post-transcriptionally limit the amount of $\Delta N p 63 \alpha$ protein, mostly by affecting $\Delta N p 63 \alpha$ proteasomal degradation rather than through a canonical miRNA/target interaction. Functionally, miR-205 is able to control the deposition of laminin-332 and its receptor integrin- $\beta 4$. Hence, pathological loss of miR-205, as widely observed in prostate cancer, may favor tumorigenesis by creating discontinuities in the BM. Here we demonstrate that therapeutic replacement of miR-205 in prostate cancer (PCa) cells can restore $\mathrm{BM}$ deposition and 3D organization into normal-like acinar structures, thus hampering cancer progression.

Cell Death and Differentiation (2012) 19, 1750-1760; doi:10.1038/cdd.2012.56; published online 4 May 2012

MicroRNAs (miRNAs) are short, non-coding RNAs that negatively regulate gene expression at the post-transcriptional level. ${ }^{1}$ By hybridizing to at least partially complementary regions on target mRNAs, miRNAs can induce mRNA degradation or translation inhibition, thus finely tuning protein expression in a variety of biological processes. ${ }^{1}$ Consequently, aberrant miRNA expression and function have been linked to the pathogenesis of human diseases, including cancer, where specific miRNAs have been proven to act as oncogenes or tumor suppressors. ${ }^{2}$

We previously showed that miR-205 is downregulated in prostate cancer (PCa) compared with adjacent non-neoplastic tissue. ${ }^{3}$ This finding was then confirmed by several independent studies (reviewed in Gandellini et al. ${ }^{4}$; Schaefer et al..$^{5}$ ), and miR-205 recognized as the best single miRNA able to correctly distinguish prostate tumor from normal tissue. ${ }^{6} \mathrm{We}$ also reported that miR-205 acts as a tumor suppressor in human prostate, as its reintroduction in $\mathrm{PCa}$ cells reverts epithelial-to-mesenchymal transition (EMT), ${ }^{3}$ thus suggesting that $m i R-205$ reduction may drive the progression toward a cell phenotype with enhanced invasive properties and favor metastasis. Accordingly, tumors from patients with lymph node dissemination show lower miR-205 expression than those from node-negative patients. ${ }^{3}$ However, evidence of a downregulation of the miRNA in clinically localized carcinomas ${ }^{4}$ suggests that loss of miR-205 in PCa may anticipate disease progression. To gain insight into this early loss of the miRNA and into the mechanisms of PCa development, we investigated the physiological role of miR-205 in normal prostate.

Prostatic epithelium is characterized by three different cell layers: (i) an outer, androgen-independent basal layer, lying on a basement membrane (BM) and characterized by the expression of high molecular weight cytokeratins $\mathrm{K} 5$ and $\mathrm{K} 14$, and the transcription factor p63; (ii) an inner secretory/luminal layer expressing androgen receptor, prostate-specific antigen, and low molecular weight cytokeratins $\mathrm{K} 8$ and $\mathrm{K} 18$; (iii) a minor cell population of neuroendocrine cells rich in serotonin-containing granules, located between secretory cells. ${ }^{7}$

It has been reported that in murine prostate miR-205 expression is higher in basal than luminal cells, ${ }^{8}$ whereas the expression pattern of miR-200 family members, despite a similar role in regulating $\mathrm{EMT},{ }^{9}$ is inverse. In addition to suggesting that functions of $m i R-205$ and miR-200 family may not completely overlap, the finding indicates that miR-205 downregulation in $\mathrm{PCa}$ may not only reflect the induction of EMT but also loss of the basal layer of epithelium.

To explain the enriched presence of miR-205 in normal basal cells, we investigated whether any transcription factor

\footnotetext{
${ }^{1}$ Department of Experimental Oncology, Fondazione IRCCS Istituto Nazionale dei Tumori, Milan, Italy; ${ }^{2}$ Department of Biomolecular Sciences and Biotechnology, University of Milan, Milan, Italy; ${ }^{3}$ Department of Pathology, Fondazione IRCCS Istituto Nazionale dei Tumori, Milan, Italy; ${ }^{4}$ Department of Radiotherapy, Fondazione IRCCS Istituto Nazionale dei Tumori, Milan, Italy and ${ }^{5}$ Prostate Program, Fondazione IRCCS Istituto Nazionale dei Tumori, Milan, Italy

*Corresponding author: N Zaffaroni, Department of Experimental Oncology, Fondazione IRCCS Istituto Nazionale dei Tumori, Via Amadeo 42, Milan 20133, Italy. Tel: +39 022390 3260; Fax: +39 022390 2692; E-mail: nadia.zaffaroni@istitutotumori.mi.it

Keywords: microRNA; prostate cancer; p63; basal layer of epithelium; basement membrane; 3D culture

Abbreviations: BM, basement membrane; ChIP, chromatin immunoprecipitation; ChIP-Seq, chromatin immunoprecipitation-sequencing; ECM, extracellular matrix; EMT, epithelial-to-mesenchymal transition; H3K27ac, H3K27 histone acetylation; H3K4me1, H3K4 histone monomethylation; H3K4me3, H3K4 histone trimethylation; ITGB4, integrin- $\beta$ 4; LAMA3, laminin- $\alpha 3$; LAMB3, laminin- $\beta 3$; LAMC2, laminin- $\gamma 2$; LNA, locked nucleic acid; miRNA, microRNA; NQO1, NAD(P)H:quinone oxidoreductase 1; PCa, prostate cancer; PIN, prostatic intraepithelial neoplasia; qRT-PCR, quantitative reverse transcriptase PCR; TSS, transcriptional start site; UTR, untranslated region

Received 26.7.11; revised 27.3.12; accepted 30.3.12; Edited by M Piacentini; published online 04.5.12
} 
exclusively expressed by basal but not luminal cells could regulate miRNA expression and focused our attention on p63. TP63 gene encodes for six different protein products, resulting from alternative splicing $-\alpha, \beta$ and $\gamma-$ at the $3^{\prime}$ end of two different pre-mRNA transcripts - amino-terminal transcriptional activation domain-containing (TAp63) and deleted p63 ( $\triangle \mathrm{Np} 63)$ - generated from two distinct promoters. ${ }^{10}$ The $\Delta \mathrm{Np} 63 \alpha$ isoform is selectively expressed at high levels in the basal cells of stratified and glandular epithelia, including prostate, and its expression decreases with differentiation. Strikingly, p63 is not expressed in most prostate carcinomas, reflecting a loss of the basal layer, and the absence of staining for p63 is almost definitively diagnostic for $\mathrm{PCa}{ }^{11}$

In this study, the presence of an interplay between $\Delta \mathrm{Np} 63 \alpha$ and miR-205 in basal cells was investigated to elucidate the physiological role of $m i R-205$ and the mechanism by which its loss may contribute to PCa development.

\section{Results}

Coregulation of miR-205 and genes involved in the maintenance of the basal phenotype. We interrogated a public dataset of gene and miRNA expression profiles (hereafter named Ambs dataset) obtained on prostatic normal and tumor tissue specimens ${ }^{12}$ to determine the genes correlated with miR-205. Positively correlated genes are supposed to be coexpressed in specific cell types and eventually participate in common pathways. Among genes positively correlated with $m i R-205$, we found a significant enrichment of gene families related to the regulation of cell morphogenesis and ectodermal development, cell-cell junctions, and cell polarity (Supplementary Table S1). Besides factors related to EMT ( $C D H 1, C D H 3)$, there were basal cytokeratins $K 5, K 14, K 15$ and $K 17$, and additional genes involved in epithelial organization, such as integrins and laminins (Supplementary Table S2). Interestingly, not only basal cytokeratins were correlated with miR-205, but miR-205 was the miRNA most strongly correlated with $K 5$ and the fifth most correlated with K14. The miRNAs most correlated with E-cadherin were instead miR-200 family members, which however did not correlate with basal cytokeratins. Overall, the findings corroborated the hypothesis that, despite a similar involvement in EMT, miR-205 could have additional functions related to maintenance of the basal compartment of epithelium.

In accord with this hypothesis, the probe set most correlated with miR-205 was 211194_s_at, which recognizes p63 (Supplementary Table S2). A significant positive correlation was also found for four other p63 probe sets (Figure 1a). In addition, miR-205 was the miRNA most correlated with the p63 probe sets 207382_at, 209863_s_at, and 211194_s_at. $P 63$, and miR-205 were both expressed at higher levels in normal than in tumor samples, thus reflecting loss of the basal cell layer from tumors. Consistently, we found a significant direct correlation between $\triangle N p 63$ mRNA and miR-205 expression in a series of paired tumor and non-neoplastic tissue specimens (Figure 1b), where the levels of both factors were generally higher in normal samples than in matched cancer tissues (Figure 1c) and correlated with the percentage of normal tissue in each sample (Supplementary Figure S1). Enrichment of miR-205 expression in basal cells of normal epithelium and loss of miR-205 in PCa were then confirmed by in situ hybridization on tissue cryosections (Figure 1d).

miR-205 and $\Delta \mathrm{Np63} \alpha$ interplay in prostate basal cells. Given the strong correlation found between p63 and miR-205, we wondered whether the miRNA could be transcriptionally regulated by $\mathrm{p} 63$ in prostate basal cells. Owing to the lack of cell lines showing a purely basal phenotype, functional investigations were performed on RWPE-1 cells. As they are derived from normal prostate tissue, ${ }^{13}$ they express both basal and luminal cytokeratins and are characterized by high levels of $m i R-205$ and $\Delta \mathrm{Np} 63 \alpha$ (Supplementary Figure S2).

To guide the search for p63 sites in prostate cells, we made use of our chromatin immunoprecipitation-sequencing (ChIP-Seq) data obtained in human $\mathrm{HaCaT}$ keratinocytes, ${ }^{14}$ where miR-205 expression is fundamental. ${ }^{15}$ We noticed two peaks of $\mathrm{p} 63$, at $\mathrm{a}-13 \mathrm{~Kb}$ and $+2 \mathrm{~Kb}$ from the recently identified $^{16}$ miR-205 transcriptional start site (TSS; Figure 2a). Search for p63 motifs in these regions highlighted three highly predicted consensus sequences in the former, and two flanking the $+2 \mathrm{~Kb}$ site (Figure $2 \mathrm{a}$ ). Furthermore, the p63 peaks were within regions enriched in positive histone modifications, that is, H3K4 monomethylation and H3K27 acetylation, in different cell types (Figure 2a). ChIP was performed in RWPE-1 cells with two anti-p63 antibodies and confirmed that p63 binding to the $-13 \mathrm{~Kb}$ peak was very strong (Figure $2 \mathrm{~b}$ ), less so for the $+2 \mathrm{~Kb}$ peak. Functionally, transcriptional regulation of miR-205 by $\mathrm{p} 63$ was verified by silencing and overexpressing $\triangle \mathrm{Np63} \alpha$ in RWPE-1 cells, which resulted, respectively, in a concomitant decrease and increase in the levels of miR-205 primary transcript and mature form (Figure 2c).

It is known that most miRNAs work in feedback loops where a transcription factor and a miRNA are mutually regulated. ${ }^{17}$ In this context, we wondered whether miR-205 was able to directly or indirectly regulate $\Delta N p 63 \alpha$. Silencing of $m i R-205$ in RWPE-1 cells resulted in a marked upregulation of $\Delta \mathrm{Np} 63 \alpha$ protein (Figure $3 a$ ), although its mRNA levels appeared to be slightly down-modulated (Figure $3 b$ ). Consistent with this finding, overexpression of miR-205 induced an appreciable reduction in the amount of $\Delta \mathrm{Np} 63 \alpha$ protein (Figure 3a), regardless of increased mRNA expression levels (Figure $3 b$ ). Such data suggested the existence of two main mechanisms by which miR-205 may reciprocate its transcriptional activation by $\Delta \mathrm{Np} 63 \alpha$, specifically a weak enhancement of p63 transcription accompanied by a major negative feedback loop effect consisting of post-transcriptional repression.

To characterize the former effect, we focused on the transcription factor ZEB1, which is implicated in EMT, tumorigenesis, and metastasis. ${ }^{18}$ ZEB1 has been validated as a miR-205 target in canine MDCK cells, where it mediates the effect of the miRNA on EMT. ${ }^{9}$ ZEB1 has been also shown to repress $\triangle N p 63$ transcription. ${ }^{19}$ In RWPE- 1 cells, silencing of miR-205 increased the amount of ZEB1 protein, thus confirming the ability of the miRNA to control ZEB1 expression (Figure 3c). In addition, silencing of ZEB1 (Supplementary Figure S3) induced up-modulation of $\triangle \mathrm{Np} 63$ transcript levels, as also obtained by overexpressing miR-205 

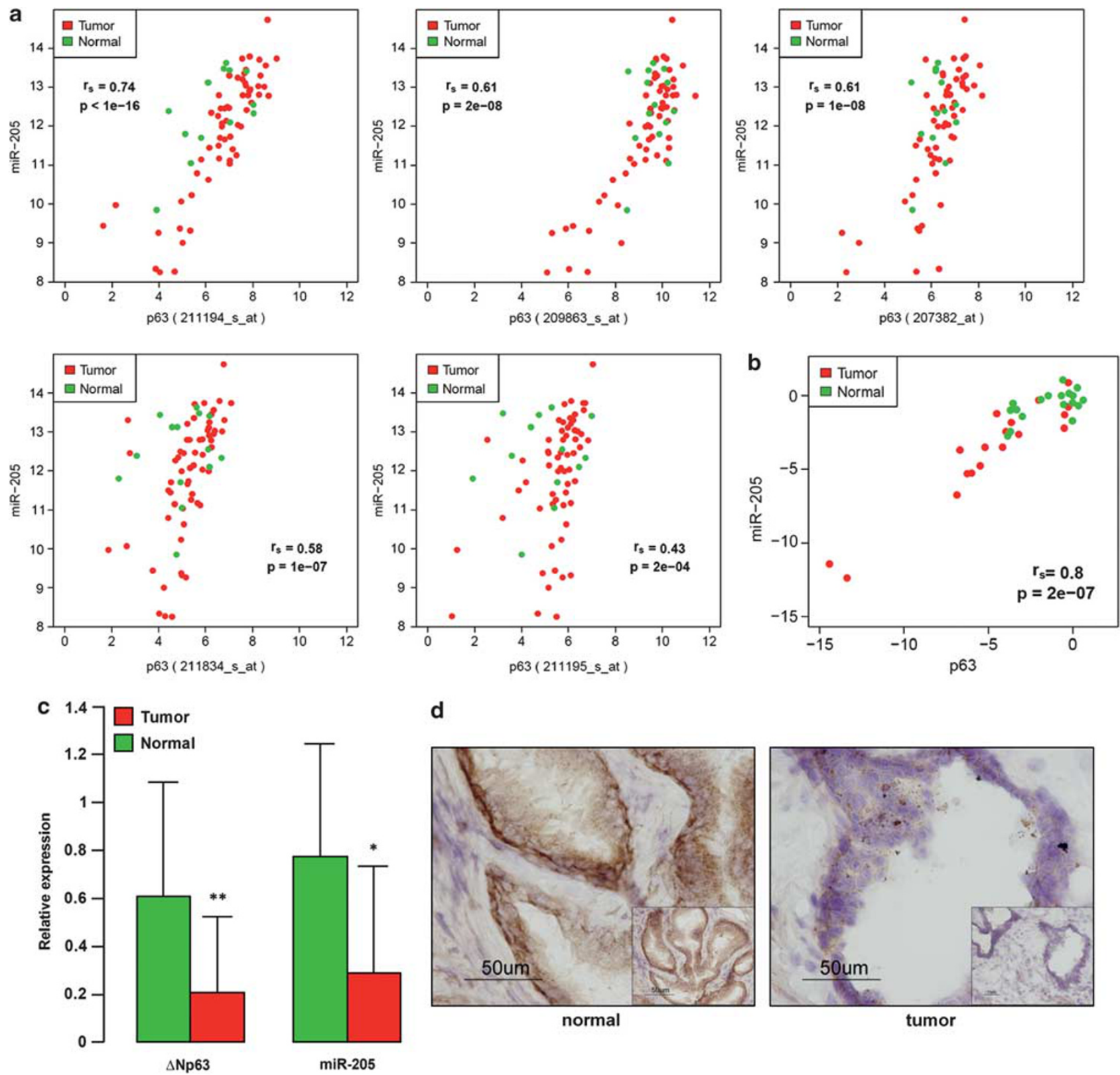

d
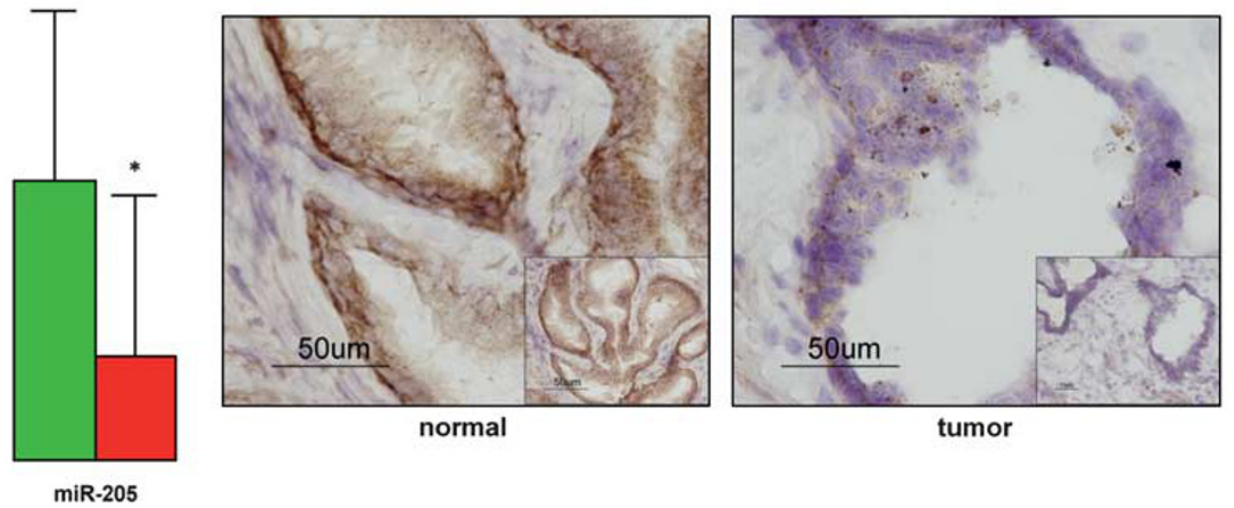

Figure 1 MiR-205 and $p 63$ expression in prostate tissues. (a) Spearman's correlation $\left(r_{s}\right)$ between miR-205 and the probe sets for p63 within Ambs dataset of clinical normal prostate (green) and prostate tumor specimens (red). (b) Correlation between p63 and miR-205 expression, as measured by qRT-PCR in our in-house series of paired normal and tumor specimens from $18 \mathrm{PCa}$ patients subjected to radical prostatectomy. (c) qRT-PCR showing higher expression of both miR-205 and $\triangle N p 63$ in normal samples with respect to matched cancer tissues. Data represent average $\Delta$ Np63 or miR-205 expression levels \pm S.D. ${ }^{*} P<0.05$; ${ }^{* *} P<0.01$. (d) In situ detection of miR-205 in a representative normal prostate tissue (left) and a tumor specimen (right), showing enriched expression of the miRNA in the basal cell layer of normal acini and loss of expression in cancer. Original magnification for insert is $\times 20$

(Figure 3b). Such findings suggested that the slight enhancement of $p 63$ transcription by miR-205 could be attributed to relief of ZEB1 repression on $p 63$ promoter.

As regards the post-transcriptional repression of p63 by miR-205, it was surprising to observe that none of the nine tested computational algorithms for miRNA target prediction found putative miR-205 binding sites in $\triangle N p 63 \alpha 3^{\prime}$ untranslated region (UTR), suggesting that miR-205 limited the amount of $\Delta \mathrm{Np} 63 \alpha$ protein mainly through an indirect mechanism, presumably by enhancing its degradation. To investigate this aspect, we exposed RWPE-1 cells to the proteasome inhibitor bortezomib and found that ectopic miR-205 expression failed to decrease $\Delta \mathrm{Np} 63 \alpha$ protein levels (Figure $3 \mathrm{~d}$ ). Rather, upon uncoupling from the proteasomal pathway, pre205-transfected cells expressed even higher $\Delta N p 63 \alpha$ protein amounts than preNeg-transfected cells (Figure 3d), thus making it more evident that miR-205 may enhance p63 transcription. In addition, miR-205 induced the 

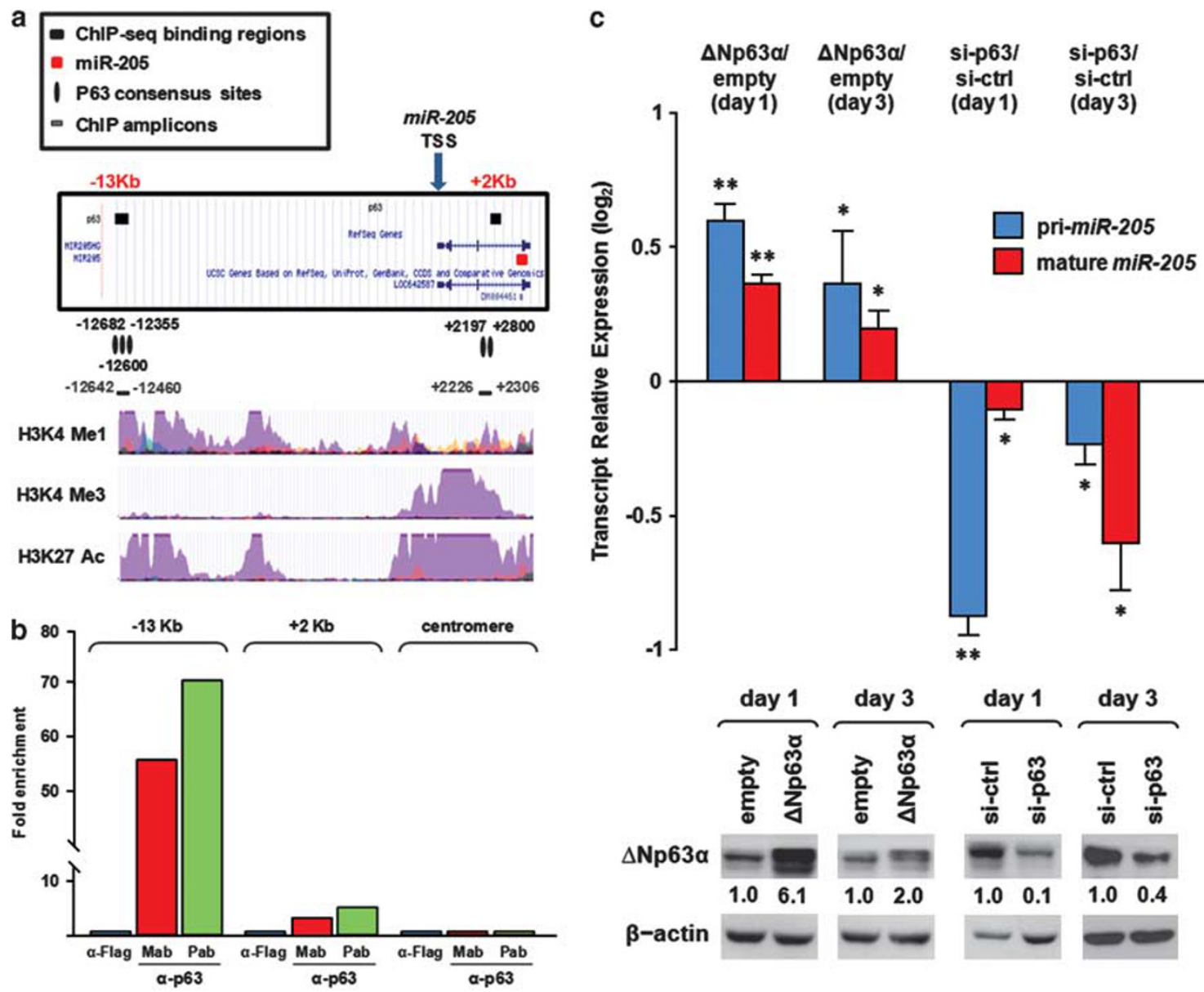

Figure 2 p63 binds miR-205 promoter and enhances miR-205 transcription in RWPE-1 cells. (a) Binding of p63 to the miR-205 locus in ChIP-Seq experiments of HaCaT keratinocytes (top). Ovals represent in silico-predicted p63 binding sites. Gray lines identify the regions at about $-13 \mathrm{~Kb}$ and $+2 \mathrm{~Kb}$ from miR-205TSS that were amplified in ChIP experiments as including or contiguous to the predicted p63-responsive elements. Positive histone modifications (H3K4me1, H3K4me3, H3K27ac) are visualized in the miR-205 locus, with respect to p63 peaks and the TSS (data from the ENCODE consortium, obtained on K562, HUVEC, H1-hESC, NHEK, GM12878, HepG2, HSMM, HMEC cell lines) (bottom). (b) Chromatin from RWPE-1 cells was precipitated with two specific anti-p63 antibodies, the mouse monoclonal 4A4 (Mab) and a rabbit polyclonal (Pab), then qRT-PCR was run to amplify regions putatively bound by p63. Flag antibody and amplification for a centromeric region were used as negative controls to normalize data. Quantification by qRT-PCR shows strong p63 enrichment in the miR-205 - 13 Kb region, less so for $+2 \mathrm{~Kb}$, as normalized for anti-flag immunoprecipitants. (c) qRT-PCR showing the levels of the primary transcript and the mature form of miR-205 in RWPE-1 cells overexpressed (using $\Delta$ Np63 $\alpha$ expression vector) or silenced (using a specific siRNA, si-p63) for $\Delta$ Np63 $\alpha$ expression. Data are reported as $\log _{2}$-transformed relative quantity with respect to the relative controls, empty vector or control siRNA (si-ctrl) (top). Western blotting showing $\Delta$ Np63 $\alpha$ expression levels in RWPE-1 cells upon transfection. Ratios of band intensities in treated samples versus controls (both normalized for $\beta$ actin as equal loading control) are reported (bottom). Overexpression of p63 resulted in enhanced transcription of miR-205, as evidenced by increased expression levels of its primary transcript, which was paralleled by an increase in the mature form. Accordingly, silencing of p63 resulted in a marked downregulation of miR-205 primary transcript starting from 1 day after transfection, which was followed by a reduction of mature miRNA levels at day $3 .{ }^{*} P<0.05 ;{ }^{* *} P<0.005$

appearance of a ladder of bands of molecular weight above $98 \mathrm{kDa}$, which also accumulated with increasing doses of bortezomib (Figure 3e), thus representing poly-ubiquitinated forms of $\Delta \mathrm{Np} 63 \alpha$. Consistently, increased ubiquitination of $\Delta \mathrm{Np} 63 \alpha$ by miR-205 was confirmed by probing anti-p63 immunoprecipitants with an anti-ubiquitin antibody (Figure 3f). Notably, the silencing of ZEB1 did not phenocopy miR-205 overexpression, as it induced an increase in $\Delta \mathrm{Np} 63 \alpha$ protein (Figure 3a), consistent with its transcriptional effect (Figure 3b).

Overall, such findings suggested that $\mathrm{p} 63$ suppression by miR-205 was ascribable to increased protein turnover, likely due to the ability of the miRNA to target genes that prevent p63 proteasomal degradation. In this context, putative
miR-205 binding sites were predicted in the $3^{\prime}$ UTR of several de-ubiquitinating enzymes, such as ubiquitin specific peptidase (USP)6, 22, 32, 35, 37, and 38, and in $\mathrm{NAD}(\mathrm{P}) \mathrm{H}$ :quinone oxidoreductase 1 (NQO1), recently shown to stabilize p63 protein by sequestering and protecting it from proteasomal degradation. ${ }^{20}$ Additional investigation would be required to discriminate which miR-205 targets are actually involved in the negative feedback loop from miR-205 to p63, however, preliminary evidence highlights the ability of miR-205 to modulate NQO1 expression (Figure $3 g$ ).

To validate the physiological relevance of the unique network involving p63 and miR-205, RWPE-1 cells were grown in different culture conditions to obtain shifts in p63 and cytokeratin expression. Strikingly, modulations of basal 
a

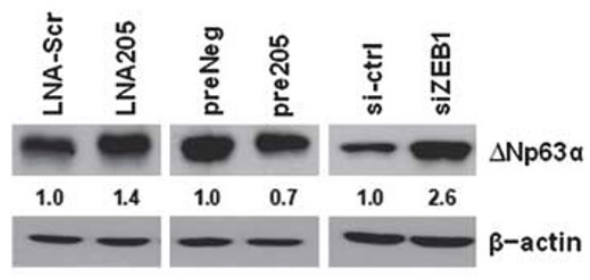

C d

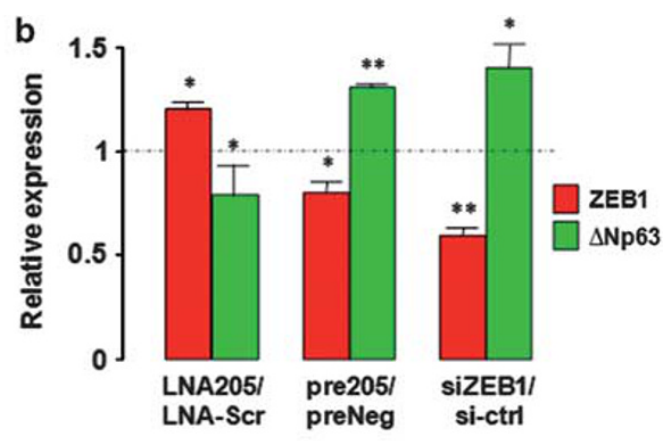

WB: $\alpha-p 63$
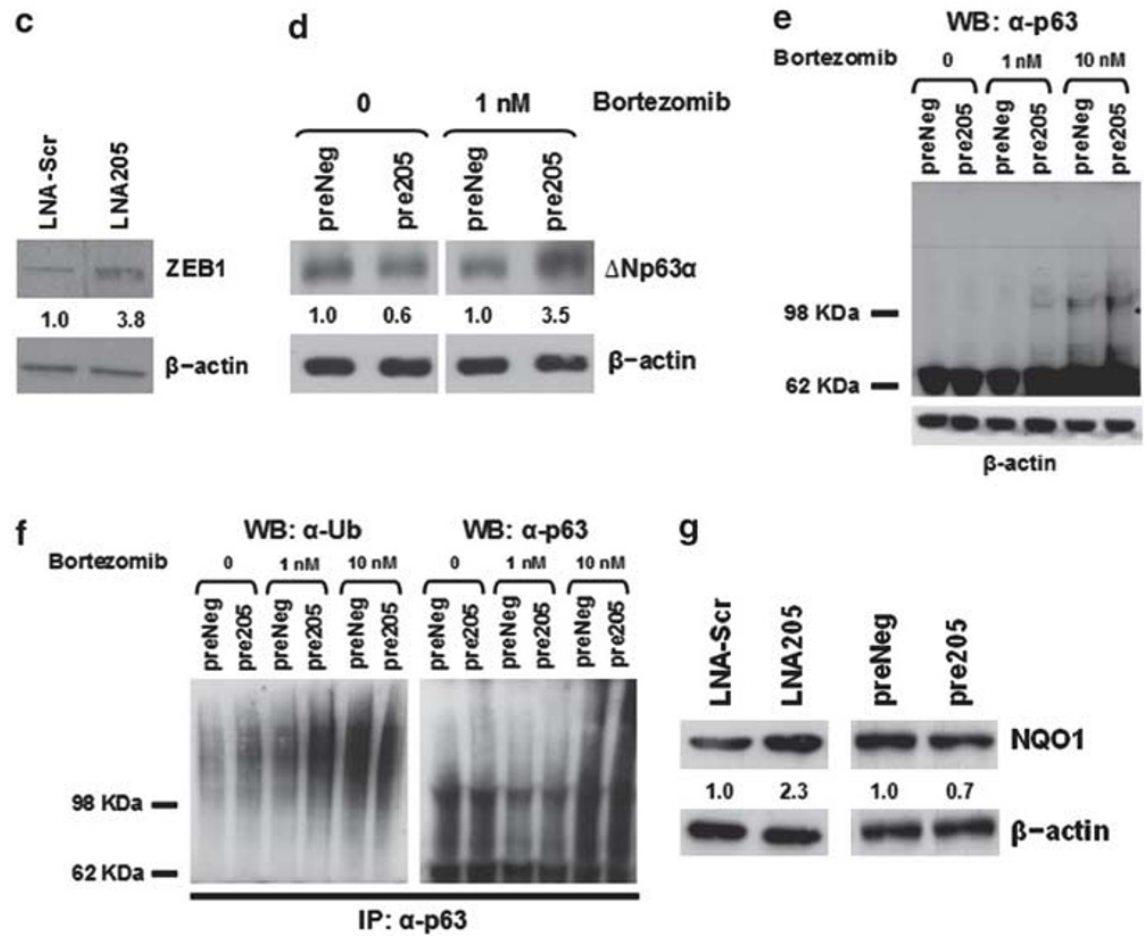

g

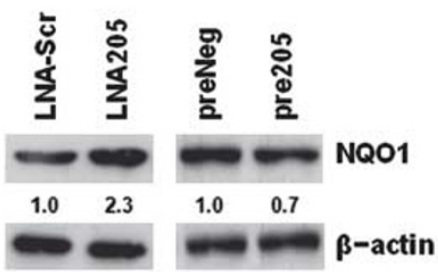

Figure 3 MiR-205 can transcriptionally enhance but post-transcriptionally repress p63 in prostatic basal cells. (a) Western blotting showing modulations of $\Delta$ Np63 $\alpha$ protein amount upon transfection of RWPE-1 cells with a LNA-modified antisense oligomer (LNA205) directed against miR-205, miR-205 synthetic precursor (pre205), and siRNA targeting ZEB1 (siZEB1). (b) Quantification of ZEB1 and $\triangle N p 63$ mRNA expression levels following miR-205 inhibition, overexpression, or ZEB1 silencing. Data are reported as relative transcript expression in LNA205-, pre205- or siZEB1-transfected cells over relative controls (LNA-Scr, preNeg, and si-ctrl). ${ }^{*} P<0.05$; ${ }^{* \star} P<0.005$. (c) Western blotting showing up-modulation of ZEB1 protein upon miR-205 silencing in RWPE-1 cells. (d) Treatment with a subtoxic concentration of bortezomib (1 nM) prevents $\Delta N p 63 \alpha$ protein reduction by pre205. (e) Overexposure of p63 blotting showing appearance of high molecular weight p63 forms both upon miR-205 overexpression and bortezomib treatment. (f) Representative IP experiment showing increased ubiquitination of $\triangle N p 63 \alpha$ by miR-205. $\alpha$-Ub: anti-ubiquitin antibody. (g) Western blotting showing that NQO1 may be a potential target of miR-205. In fact, NQ01 levels are increased or reduced in RWPE-1 cells upon miR-205 silencing or overexpression, respectively

markers were paralleled by correlated variations in miR-205 expression, thus supporting both the functional relationship between $\Delta \mathrm{Np63}$ and $\mathrm{miR}-205$ and the actual involvement of miR-205 in maintenance of the basal phenotype (Figure 4).

Functional role of miR-205 in basal cells. The specific role of miR-205 was investigated by profiling gene expression upon its depletion in RWPE-1 cells. A gene ontology enrichment analysis conducted on differentially expressed genes showed over-representation of functional families related to the extracellular region, cell cortex, and proteinaceous extracellular matrix (ECM) (Supplementary Figure S3), suggesting that miR-205 could mainly regulate the interaction between basal cells and the BM. In this context, we focused on laminin-332, the major adhesive component of prostatic BM. Composed of three subunits $(\alpha 3, \beta 3$, and $\gamma 2)$, it is a ligand for at least two integrins expressed by epithelial cells, $\alpha 3 \beta 1$ and $\alpha 6 \beta 4$. $^{21,22}$

In Ambs dataset, expression of $L A M A 3$, LAMB3, LAMC2, and ITGB4 was directly correlated with that of miR-205, suggesting a functional relationship. Accordingly, mRNA levels of all components of the laminin-332/integrin- $\beta 4$ complex decreased in RWPE-1 cells silenced for miR-205, as evidenced by gene expression profiles and quantitative reverse transcriptase PCR (qRT-PCR; Figure 5a). Consistent with this observation, the amounts of laminin- $\beta 3$, laminin- $\gamma 2$, and integrin- $\beta 4$ proteins in the whole extracts (Figure $5 \mathrm{~b}$ ) and in the media (Figure 5c) obtained from RWPE-1 cells were 
a
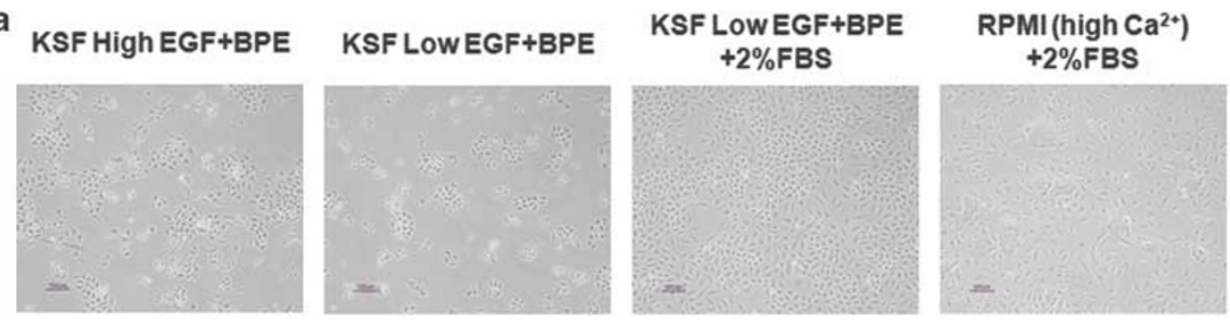

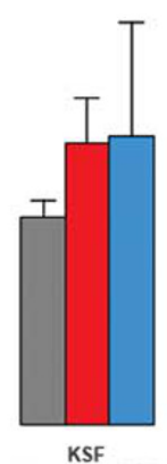

Low EGF+BPE

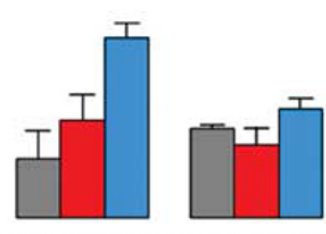

KSF Low EGF+BPE RPMI (high Ca ${ }^{2 *}$

\begin{tabular}{|c|c|c|c|}
\hline Correlation & $\mathrm{p} 63$ & $\mathrm{~K} 14$ & $\mathrm{miR}-205$ \\
\hline p63 & 1.00 & 0.92 & 0.70 \\
\hline K14 & 0.92 & 1.00 & 0.92 \\
\hline miR-205 & 0.70 & 0.92 & 1.00 \\
\hline
\end{tabular}

$+2 \%$ FBS

Figure 4 Coordinate modulation of miR-205 and p63 during in vitro differentiation of RWPE-1 cells. (a) Representative phase-contrast micrographs of RWPE-1 cells grown in different culture conditions known to induce shifts in cytokeratin expression. High: $5 \mathrm{ng} / \mathrm{ml}$ epidermal growth factor (EGF) and $50 \mu \mathrm{g} / \mathrm{ml}$ bovine pituitary extract (BPE), low: $1.25 \mathrm{ng} / \mathrm{ml} \mathrm{EGF}$ and $12.5 \mu \mathrm{g} / \mathrm{ml} \mathrm{BPE}$. (b) Quantification of $\Delta N p 63, K 14$, and miR-205 expression levels by qRT-PCR in RWPE-1 cells cultured in media. Data are reported as relative expression with respect to cells grown in standard conditions (high EGF and BPE concentration). The table on the right shows calculated correlation coefficients between $\triangle N p 63, K 14$, and miR-205. Specifically, lowering of EGF and BPE concentration in keratinocyte serum-free (KSF) medium resulted in increased $\Delta N p 63$ and $K 14$ expression levels, whereas addition of serum or culturing in high calcium RPMI medium reduced them

reduced following miR-205 depletion. Conversely, miR-205 overexpression increased laminin mRNA and protein levels, thus confirming that the miRNA is involved in the regulation of BM deposition by prostate basal cells (Figures $5 \mathrm{a}-\mathrm{c}$ ). These results were validated by laminin- $\gamma 2$ immunofluorescence (Figure 5d).

As ZEB1 has been shown to repress the transcription of LAMA3, LAMB3, LAMC2, and ITGB4, ${ }^{23,24}$ we checked whether miR-205 might enhance laminin production by down-modulating ZEB1. Strikingly, silencing of ZEB1 relieved transcriptional repression on only $\angle A M A 3$ and $\angle A M B 3$ genes and instead induced downregulation of LAMC2 and ITGB4 mRNAs and proteins (Figures 5a-d), as previously reported by Spaderna et al. ${ }^{24}$ Overall, these findings suggested that ZEB1 may not be the crucial mediator of miR-205 effect on laminins.

Given the reciprocal interplay between miR-205 and p63, we wondered whether $\Delta \mathrm{Np} 63 \alpha$ could also stimulate $\mathrm{BM}$ deposition and whether it could be specifically mediated by miR-205. In RWPE-1 cells, $\Delta \mathrm{Np63 \alpha}$ overexpression increased protein (Figure 6a) and mRNA levels (Figure 6b) of all laminin-332/integrin- $\beta 4$ complex subunits, although to a lesser degree than miR-205 overexpression. This effect appeared to be at least in part dependent on p63-induced up-modulation of miR-205 (Figure 2c), as it was reverted by locked nucleic acid (LNA)205 (Figure 6).

miR-205 stimulates BM deposition by $\mathrm{PCa}$ cells. $\mathrm{PCa}$ is characterized by disruption of the acinar architecture of epithelium, with loss of the basal layer. ${ }^{10}$ As a consequence, $\mathrm{PCa}$ cells have a predominantly luminal phenotype and express neither $\mathrm{p} 63^{10}$ nor $\mathrm{miR}-205 .{ }^{3}$ We wondered whether the capability of miR-205 to regulate BM deposition could be at least partially retained in p63-deficient PCa cells. We found that restoration of miR-205 in DU145 and PC-3 cells was sufficient to increase the expression levels of all the components of the laminin-332 complex (Figures $5 \mathrm{~d}$ and e and Supplementary Figure S4), even though p63 mRNA and protein levels remained undetectable (data not shown). Conversely, $\Delta \mathrm{Np63} \alpha$ increased protein (Figure 6a) and mRNA levels (twofold) of only integrin- $\beta 4$, an effect that was not reverted by LNA205. Notably, miR-205 expression remained undetectable in $\Delta \mathrm{Np} 63 \alpha$-expressing $\mathrm{PC}-3$ cells (data not shown), thus confirming the strong epigenetic repression of the miRNA found in these cells ${ }^{25}$ and showing that, in the absence of miR-205, p63 cannot enhance production of the whole laminin-332 complex. The retained ability of $\mathrm{p} 63$ to induce integrin- $\beta 4$ may rely on a direct transcriptional activation, as reported by Carroll et al. ${ }^{26}$ In this context, integrin- $\beta 4$ expression levels were the least affected by $m i R$-205 silencing also in $\Delta$ Np63 $\alpha$-overexpressed RWPE1 cells (Figure 6).

To assess the effect of laminin re-expression by miR-205 on the growth of $\mathrm{PCa}$ cells in a three-dimensional landscape, invasive PC-3 cells were grown in growth factor-reduced Matrigel. PreNeg-transfected cells mainly grew as nonpolarized masses, which turned into a stellate-invasive phenotype from day 7 (Figure 7a), as also described by 
a

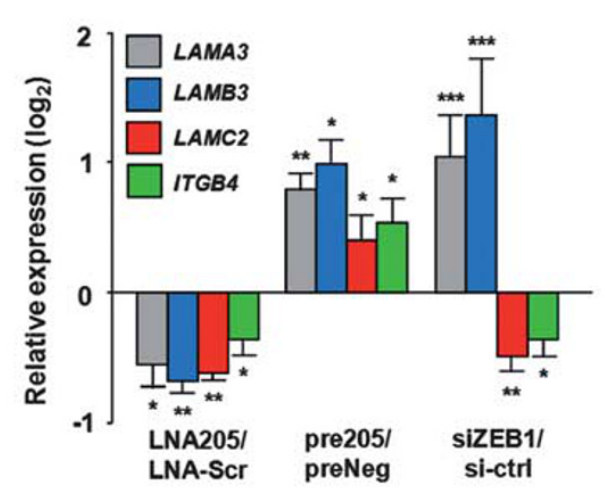

b

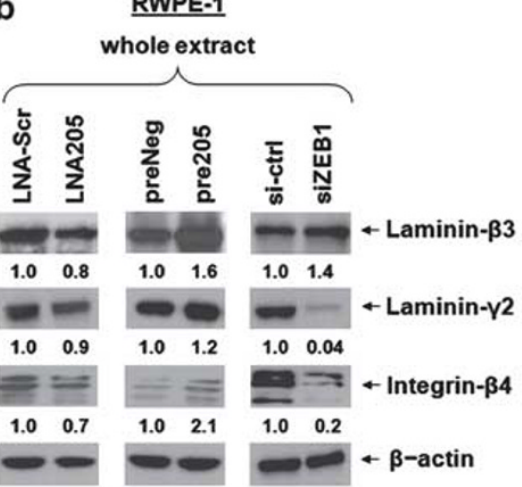

c
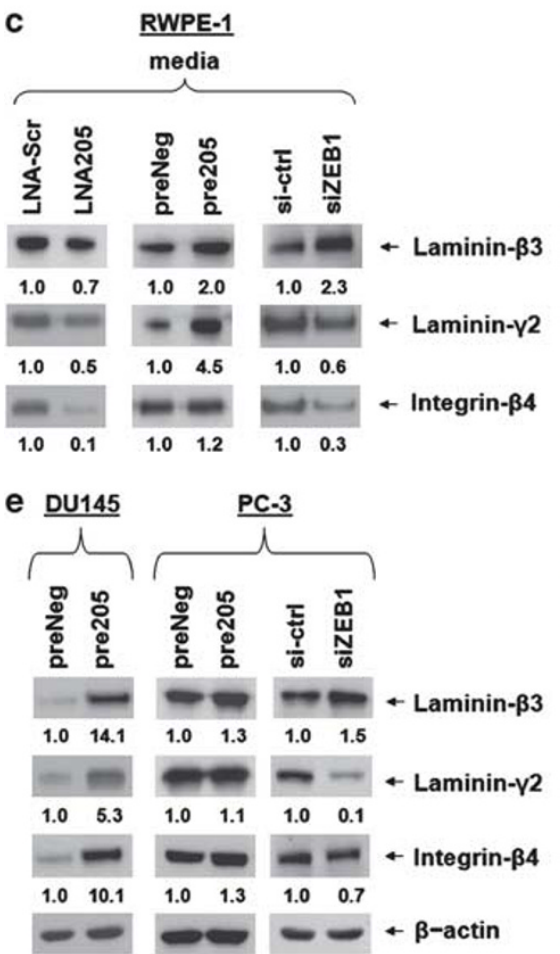

d

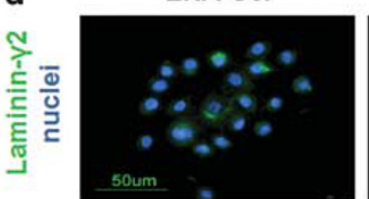

preNeg

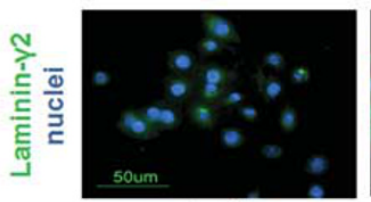

si-ctrl

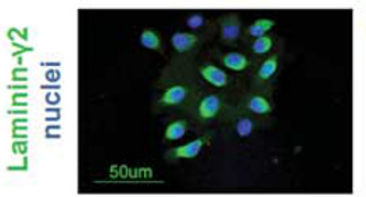

preNeg

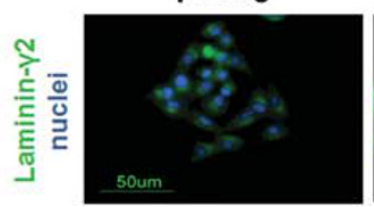

LNA205

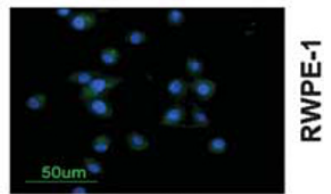

pre205

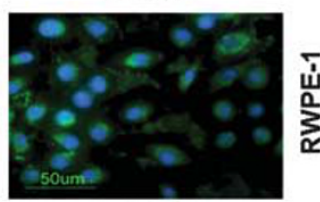

SIZEB1

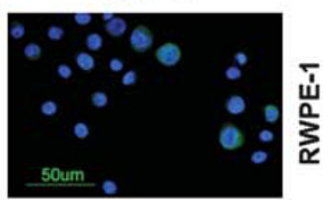

pre205

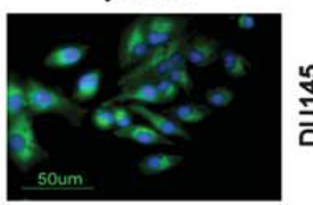

Figure 5 miR-205 is able to regulate the expression of laminin-332 and its receptor integrin- $\beta 4$. (a) qRT-PCR assessment of mRNAs levels of LAMA3, LAMB3, LAMC2, and ITGB4 in RWPE-1 cells upon modulation of miR-205 expression or silencing of ZEB1. Data are reported as $\log _{2}$-transformed relative quantity with respect to the relative controls. ${ }^{*} P<0.05$; ${ }^{* *} P<0.005$; ${ }^{* * *} P<0.0005$. (b) Western blotting showing laminin- $\beta 3$, laminin- $\gamma 2$, and integrin- $\beta 4$ expression levels in the whole extracts from RWPE-1 cells modulated for miR-205 expression or silenced for ZEB1. (c) Western blotting showing laminin- $\beta 3$, laminin- $\gamma 2$, and integrin- $\beta 4$ expression levels in the media from RWPE- 1 cells modulated for miR-205 expression or silenced for ZEB1. (d) Immunofluorescence showing the effect of modulating either miR-205 or ZEB1 on laminin- $\gamma 2$ deposition by RWPE-1 cells. The effect on laminin- $\gamma 2$ production by DU145 cancer cells restored for miR-205 expression is also reported. (e) Western blotting showing that miR-205 ectopic expression is sufficient to induce the expression of the components of laminin-332/integrin- $\beta 4$ complex in PCa cells. However, silencing of ZEB1 can only up-modulate laminin- $\beta 3$ expression, besides downregulating laminin- $\gamma 2$ and integrin- $\beta 4$

Härmä et al. ${ }^{27}$ However, miR-205 transient re-expression inhibited the formation of invadopodia and allowed PC-3 cells to organize as acinar structures surrounded by an intact BM, as assessed by microscopy and immunofluorescence (Figure 7). Such findings suggested that the enhancement of laminin-332/integrin- $\beta 4$ production by $m i R-205$ was sufficient to restore a normal-like epithelial organization of $\mathrm{PCa}$ cells and inhibit invasion through the ECM. Again, silencing of ZEB1 in PC-3 cells (Supplementary Figure S3) did not completely phenocopy the rearrangements induced by miR-205, as it failed to induce up-modulation of all the components of the laminin complex (Figure 5e). Accordingly, suppression of ZEB1 only partially reverted the invasive phenotype of PC-3 cells grown in Matrigel, as it just retarded the sprouting of invadopodia, without evidence of acinar organization (Figure 7).

\section{Discussion}

Forming a continuous layer in human prostate, basal cells are the presumed progenitor cells of prostatic epithelium and exclusively express $\Delta \mathrm{Np} 63,{ }^{7}$ which is required for key events 

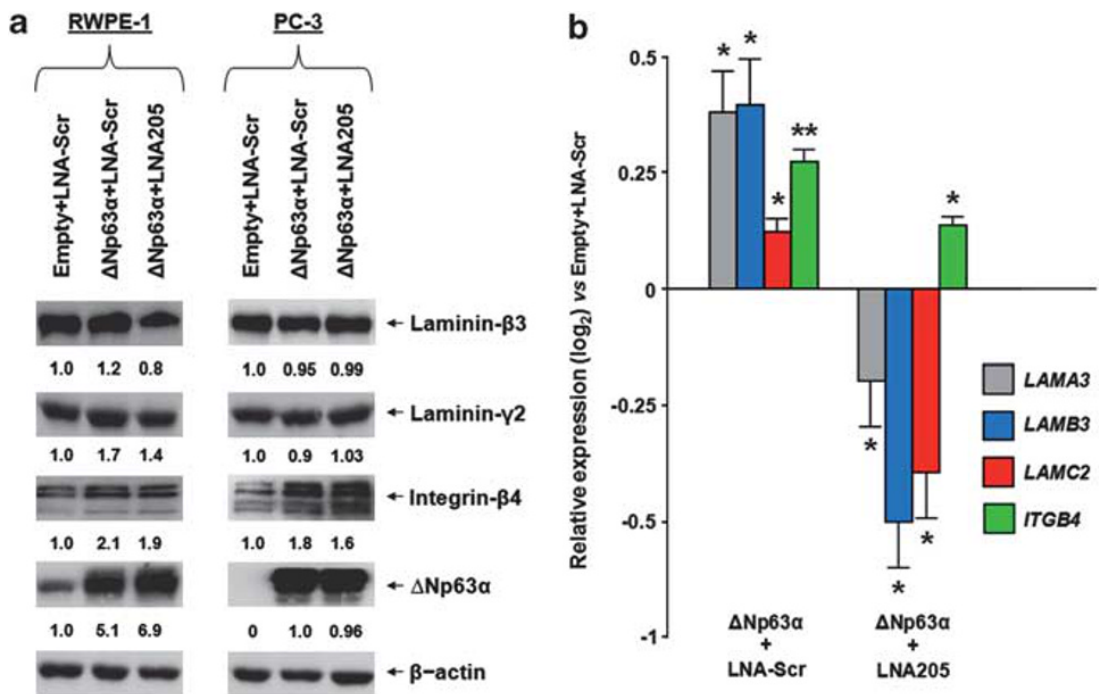

Figure 6 Induction of laminin-332/integrin- $\beta 4$ complex by $\Delta N p 63 \alpha$ is at least in part dependent on miR-205. (a) Western blotting showing laminin- $\beta 3$, laminin- $\gamma 2$, integrin$\beta 4$ and p63 expression levels in the whole extracts from RWPE-1 or PC-3 cells overexpressed for $\Delta$ Np63 $\alpha$ in combination or not with miR-205 inhibitor. (b) qRT-PCR assessment of mRNAs levels of LAMA3, LAMB3, LAMC2 and ITGB4 in RWPE-1 cells overexpressed for $\triangle N$ p63 $\alpha$ in combination or not with miR-205 inhibitor. Data are reported as $\log _{2}$-transformed relative quantity with respect to the cells transfected with the combination of empty vector and LNA-Scr. ${ }^{*} P<0.05 ;{ }^{*} P<0.01(P$-values calculated with respect to empty + LNA-Scr for $\Delta$ Np63 $\alpha+$ LNA-Scr-transfected cells and with respect to $\Delta$ Np63 $\alpha+$ LNA-Scr for $\Delta$ Np63 $\alpha+$ LNA205-transfected cells)

in prostate development, including lineage commitment and differentiation. ${ }^{28,29} \Delta \mathrm{Np63}$ is also involved in regulating deposition of the BM, a layer of specialized ECM that surrounds normal glands and separates epithelial cells from underlying mesenchymal tissues, thus providing a biophysical support as well as a hub to regulate cell-cell and cell-protein interactions. ${ }^{21,29-31}$

Starting from the evidence that miR-205 expression is enriched in murine prostatic basal cells, ${ }^{8}$ we identified an interplay between $\Delta \mathrm{Np} 63 \alpha$ and $m i R-205$ in human basal cells, where $\Delta N p 63 \alpha$ is able to enhance miR-205 transcription, whereas the miRNA can post-transcriptionally limit the amount of p63 protein (Figure 8). Apparently, this negative feedback loop relies on differential p63 proteasomal degradation rather than on a canonical miRNA/target interaction, as no binding sites for miR-205 were predicted in the $3^{\prime} U T R$ of $\triangle N p 63 \alpha$. However, when we extended the search to other regions in $\triangle N p 63 \alpha \mathrm{mRNA}$, a putative miR-205 site was found in $5^{\prime} U T R$, thereby suggesting that $m i R-205$ might also induce $\Delta \mathrm{Np} 63 \alpha$ silencing by the classical miRNA pathway, even through a non-canonical interaction.

The bi-univocal regulation between miR-205 and p63 guarantees their coordinate expression in prostate basal cells, as evidenced by the analysis of clinical series (Figure 1) and functionally validated in a differentiation assay (Figure 4). It is noteworthy that coexpression of miR-205 and p63 is not specific for prostatic tissue, as miR-205 has been also detected in the basal/myoepithelial layers of human mammary gland $^{32}$ and in p63-positive tumors, such as squamous cell lung cancer. In this context, high expression of miR-205 has been proposed as a marker of squamous histology compared with adenocarcinoma, ${ }^{33}$ but an oncogenic role for the miRNA has not been proved. Overall, the widespread coexpression of miR-205 and p63 in basal cells from glandular and stratified epithelia underscores the participation of miR-205 in p63- regulated pathways that are fundamental for the maintenance of epithelial structure.

We found that depletion of only miR-205 did not convert RWPE-1 cells into full-blown luminal cells, thus suggesting that additional arrangements (such as the concomitant downregulation of $\mathrm{p} 63$ and up-modulation of miR-200 family members) are necessary to complete transdifferentiation of basal into secretory cells. However, silencing of miR-205 was sufficient to reduce the secretion of all subunits of laminin-332 (the first ECM protein to be synthesized during embryogenesis and thought to have a crucial role in morphogenesis and organogenesis ${ }^{22}$ ) and integrin- $\beta 4$. As laminin-332/integrin $\alpha 6 \beta 4$ complexes are critical to connect the ECM to intermediate filaments within hemidesmosomes, ${ }^{22}$ miR-205 may be involved in stably anchoring prostatic basal cells to basal lamina, thus controlling normal prostate morphogenesis and tissue integrity.

Loss of cell polarity and cell:BM adhesion has been observed in many carcinomas including invasive $\mathrm{PCa}$ and advanced prostatic intraepithelial neoplasia (PIN), which is generally accepted as a precursor of invasive cancer. ${ }^{10,11,31}$ One of the hallmarks of PIN is indeed the focal attenuation of the basal cell layer, which is completely lost in even low-grade invasive carcinoma. As the lack or discontinuity of BM structures is a prerequisite for tumor cell invasion into the interstitial space and metastatic dissemination, BM maintenance represents a barrier against cancer development and progression. On the basis of our data, pathological loss of miR-205 in basal cells may result in a reduced expression of BM components and pave the way for the development of PIN and invasive cancer, which are indeed characterized by loss of integrin- $\beta 4$ and laminin-332, ${ }^{22,31}$ both regulated by the miRNA. In this context, it is worth mentioning that the $\mathrm{PCa}$ origin is still unclear. ${ }^{34}$ The evidence that $\mathrm{PCa}$ is characterized by a predominantly luminal phenotype convinced many 

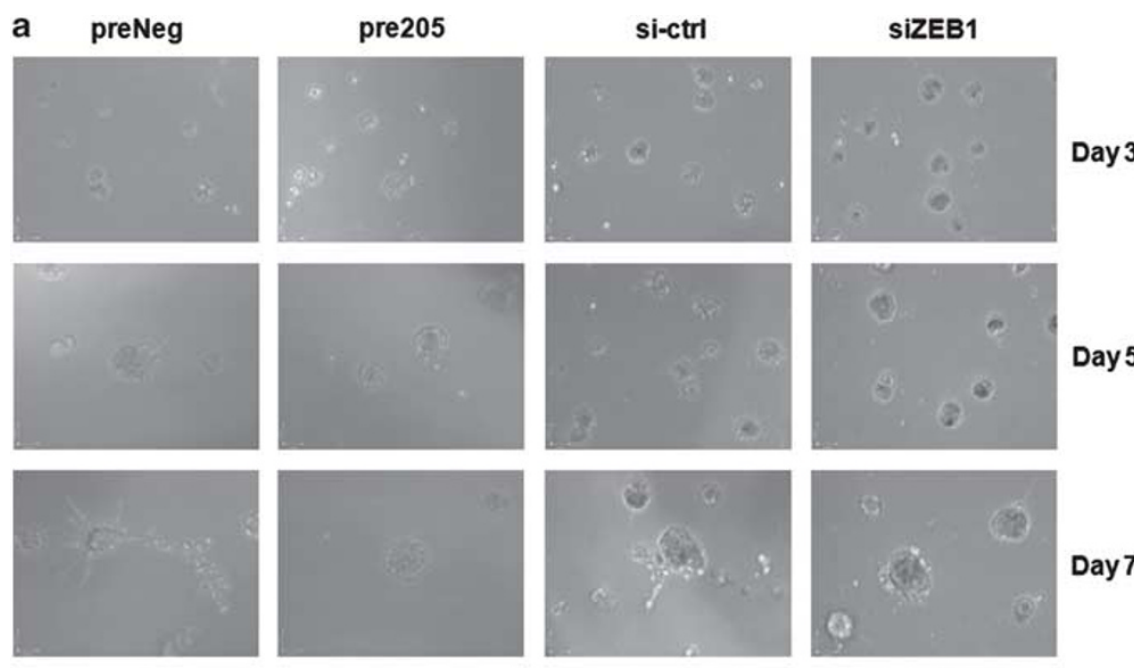

Day 7
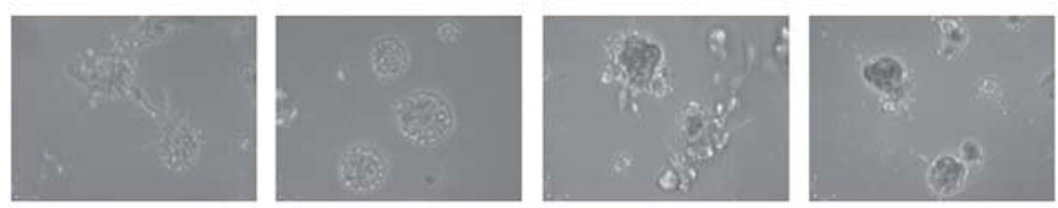

Day 9

b preNeg
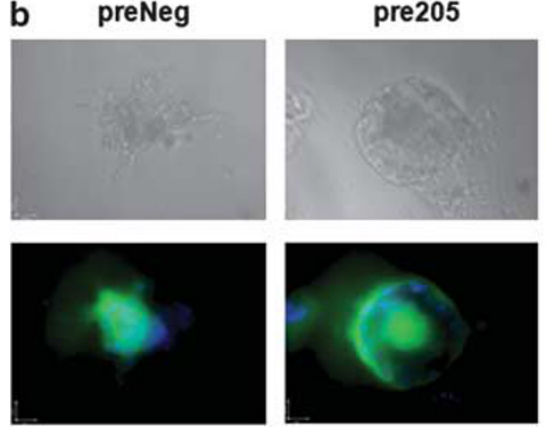
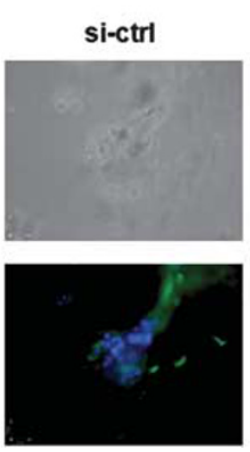

SiZEB1

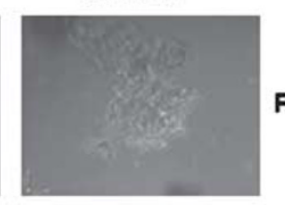

Phase contrast

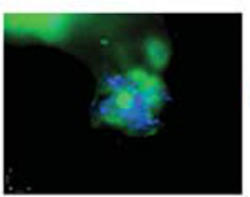

Integrin- $\beta 4$

nuclei

Figure 7 miR-205 allows prostate cancer cells to reorganize into normal-like 3D structures. (a) Time course assessment of three-dimensional growth of PCa cells in Matrigel. Micrographs are provided for cells overexpressed for miR-205 or silenced for ZEB1, with relative controls. (b) Immunofluorescence on 3D cultures showing that miR-205 ectopic expression is sufficient to revert invasive phenotype of PC-3 cells into a normal-like acinar morphology, where cell/ECM contacts stain for laminin receptor integrin- $\beta 4$. However, silencing of ZEB1 does not restore normal morphology

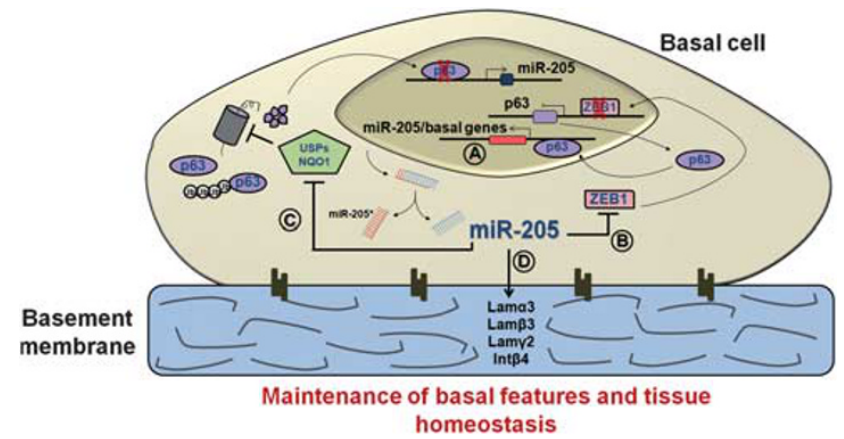

Figure 8 Theoretical miR-205/p63/laminin network in prostate basal cells. The drawing summarizes the network involving $\Delta N p 63$ and $m i R-205$, where $p 63$ can enhance miR-205 transcription (A), miR-205 transcriptionally regulates p63 by repressing ZEB1 $(\mathrm{B})$, and miR-205 indirectly reduces $\Delta$ Np63 protein amount by increasing its proteasomal degradation (C). Functionally, miR-205 is able to regulate laminin-332 deposition, thus playing a pivotal role in the maintenance of tissue integrity $(D)$ scientists that tumor cells might arise as a consequence of mutations in luminal cells. Low expression of miR-205 (and p63) in PCa cells may hence just reflect their luminal origin. However, Lawson et al. ${ }^{35}$ recently demonstrated that a phenotypically luminal PCa may also arise from basal cells upon constitutive activation of oncogenic signaling pathways. Starting from this evidence, we cannot exclude that pathological miR-205 loss in prostate basal cells may favor malignant transformation. In our hands, however, the sole depletion of miR-205 in RWPE-1 cells did not result in enhanced cell growth in vitro (data not shown).

miR-205 overexpression in cancer cells was sufficient to enhance expression of different subunits of laminin-332, thus indicating that its capability to modulate BM assembly is at least in part independent of $\Delta \mathrm{Np} 63 \alpha$. This aspect is of particular interest in the cancer context, as it would suggest that $m i R-205$ replacement therapy can be envisaged not only to increase cell-cell adhesion and reduce intrinsic invasion of 
PCa cells, ${ }^{3}$ but also to counteract the loss of the basal cell layer. Strikingly, in 3D-culture experiments, miR-205 indeed restored normal morphogenesis of invasive PCa cells, likely due to increased deposition of BM components. Accordingly, Wang et al. ${ }^{36}$ showed that invasive MDA-MB-231 breast cancer cells can be induced to form morphologically normal structures, a phenomenon called 'phenotypical reversion', upon treatment with inhibitors of integrin- $\beta 1$ or epidermal growth factor receptor.

Overall, our data show that miR-205 participates in a network involving $\Delta \mathrm{Np} 63 \alpha$ and ZEB1, which seems to be essential for maintenance of the basal cell layer of prostatic epithelium. Specifically, miR-205 is able to modulate BM deposition (Figure 8), thus being presumably required for prostate morphogenesis and to guarantee tissue integrity. As a consequence, pathological loss of miR-205 may favor tumorigenesis by creating discontinuities in the BM. From a therapeutic point of view, miR-205 replacement in PCa cells may both revert EMT and increase BM deposition, thus ultimately hampering PCa progression.

\section{Materials and Methods}

See Supplemental Materials and Methods for the description of experimental models, qRT-PCR, microarray analysis, primers, and antibodies used.

Ambs dataset analysis. Normalized miRNA and mRNA data were downloaded from GEO repository (http://www.ncbi.nlm.nih.gov/geo), with GEO submission accession number GSE8126 and GSE6956, respectively. Gene expression data were not further processed, whereas miRNA data were filtered, discarding mouse probes, and multiple probes were averaged. Data were analyzed by using R software (http://www.R-project.org) to determine the genes correlated with miR-205. Positively correlated genes were functionally classified using DAVID (http://david.abcc.ncifcrf.gov/) public software, and gene ontology enrichment analysis was performed using Affymetrix (Santa Clara, CA, USA) gene chip HG-U133A 2.0 as background.

In situ hybridization. In situ hybridization for miR-205 on human prostate tissue cryosections $(4 \mu \mathrm{m})$ was performed using miRCURY LNA microRNA ISH Optimization kit ( $\# 90000$, Exiqon, Vedbaek, Denmark), according to the manufacturer's instructions. Briefly, $40 \mathrm{nM}$ of $5^{\prime}$-biotin labeled miRCURY LNA miRNA detection probe for human miR-205 (\#18099-03, Exiqon) was hybridized onto glass slides at $55^{\circ} \mathrm{C}$ for $2 \mathrm{~h}$. Dako REAL Detection System Peroxidase/ $\mathrm{DAB}+$ kit (K5001, Dako, Glostrup, Denmark), designed for the detection of biotinylated probes, was then used according to the manufacturer's instructions. Nuclei were counterstained with hematoxylin. Images were acquired at room temperature in oil immersion by a Nikon Eclipse Ti equipped with Hamamatsu ORCA-R2 camera and Nikon PlanFluor $40 \times / 1.30$ oil or Nikon PlanAPO VC $20 \times 10.75$, using Volocity 5.4 software (PerkinElmer, Walthan, MA, USA), then processed with Adobe Photoshop Image Reader 7.0.

Chromatin immunoprecipitation. The procedure for ChIP was essentially as described in Testa et al. ${ }^{37} \mathrm{~A}$ total of $3-5 \times 10^{6} \mathrm{RWPE}-1$ cell equivalents of chromatin were immunoprecipitated with $10-15 \mu \mathrm{g}$ of anti-p63 or anti-flag control antibodies. After reversal of formaldehyde cross-linking, RNase A and proteinase $\mathrm{K}$ treatments, input and IP-enriched DNAs were used to perform qRT-PCR to amplify regions putatively bound by $p 63$. A genomic region (centromeric satellite 11) was also amplified as a negative control. A Biorad (Hercules, CA, USA) MyIQ single color thermal cycler and a SYBR Green PCR Master mix were used for qRT-PCR. Specificity of products was monitored with a heat dissociation curve. For ChIP, fold enrichment was calculated with the $2^{-\Delta \Delta C t}$ method, where the $\mathrm{Ct}$ represented the threshold cycles of the input, the specific antibody and the negative antibody. A detailed description of antibodies and region-specific primers used in ChIP experiments can be found in Supplemental Materials and Methods.

The presence and position of putative p63 binding sites within precipitated regions was predicted using the CONSITE algorithm (http://www.phylofoot.org/), employing the matrix reported in Ortt and $\operatorname{Sinh}^{38}$ and setting a cutoff $\geq 0.85$.

Cell-based experiments. Cells seeded at the appropriate density were transfected for $4 \mathrm{~h}$ with $100 \mathrm{nM}$ LNA-modified antisense oligomers, $20 \mathrm{nM}$ miRNA precursors, $25 \mathrm{nM}$ siRNAs or $0.5 \mathrm{ng} / \mu \mathrm{l} \Delta \mathrm{Np} 63 \alpha$ expression/empty vector using Lipofectamine2000 (Invitrogen/Life Technologies, Carlsbad, CA, USA), according to the manufacturer's instructions, and processed for $48-72 \mathrm{~h}$ after transfection. For siRNA sequences, and LNA and miRNA precursors refer to Supplemental Materials and Methods.

miRNA target prediction. The presence of putative miR-205 binding sites in a given mRNA was predicted using MiRWalk (http://www.ma.uni-heidelberg.de/ apps/zmf/mirwalk), which performs a comparison of the information produced by nine established miRNA target prediction programs, and PITA (http://genie.weizmann.ac.il/pubs/mir07/index.html) online tools. Only targets simultaneously predicted by at least five programs were considered as reliable.

Immunoblotting analyses. For immunoblotting, $30 \mu \mathrm{g}$ of cell lysates or $12.5 \mu \mathrm{l}$ of cultured media (after removing of cell debris and concentration with Amicon Ultra-4, Centrifugal Filter Device, Millipore, Billerica, MA, USA) were fractioned by SDS-PAGE and transferred onto Hybond nitrocellulose membranes (GE Healthcare Europe GmbH, Pollards Wood, UK). Equal protein loading was verified by Ponceau staining. Filters were blocked in PBS-Tween-20/5\% skim milk and probed with specific antibodies, which were visualized by SuperSignal West PICO chemiluminescent detection system (Pierce Biotechnology, Rockford, IL, USA). Densitometric analysis has been carried out using Vision Works LS software (UVP, Upland, CA, USA) and $\beta$-actin was used as equal protein loading control for cell lysates.

Immunoprecipitation. For assessment of the ubiquitinated form of $p 63$, precleared, detergent-solubilized cell extracts $(100 \mu \mathrm{l})$ were immunoprecipitated with the anti-p63 $4 \mathrm{~A} 4$ antibody $(200 \mathrm{ng} / \mathrm{ml})$ for $16 \mathrm{~h}$ at $4{ }^{\circ} \mathrm{C}$, with precipitation of the immune complex by addition of $50 \mu$ of a $50: 50$ protein A slurry.

Immunofluorescence on 2D cultures. For immunofluorescence analyses, cells grown on glass coverslips were fixed with $4 \%$ formaldehyde, permeabilized with $70 \%$ cold ethanol, then probed with specific primary antibodies for $1 \mathrm{~h}$ and with Alexa Fluor488-labeled (Life Technologies) secondary antibodies for $1 \mathrm{~h}$ at room temperature. Nuclei were counterstained with DAPI. Images were acquired at room temperature in oil immersion by a Nikon Eclipse Ti equipped with Hamamatsu ORCAR2 camera and Nikon PlanFluor $40 \times / 1.30$ oil using Volocity 5.4 software (PerkinElmer), then processed with Adobe Photoshop Image Reader 7.0.

3D cultures. 3D cultures were prepared as described by Tyson et al., ${ }^{39}$ with few modifications. Briefly, $10 \mu \mathrm{l}$ of thawed growth factor-reduced Matrigel (E6909, Sigma, St. Louis, MO, USA) was added to each well of $\mu$-slides angiogenesis plates (Ibidi, Munchen, Germany). After a 1 -h incubation at $37^{\circ} \mathrm{C}, 1000 \mathrm{cell} / \mathrm{sell}$ were added to $50 \mu \mathrm{l}$ of culture medium containing $2 \%$ Matrigel. Medium was then replaced every 3 days and cell morphology was checked in a time-lapse humidified chamber at $37^{\circ} \mathrm{C}$ under a Nikon Eclipse Ti equipped with Hamamatsu ORCA-R2 camera and Nikon PlanAPO VC $20 \times 10.75$ using Volocity 5.4 software. $3 \mathrm{D}$ reconstitutions were performed by Volocity 5.4 software without setting alterations. For immunofluorescence analyses, 3D cultures were fixed in $4 \%$ paraformaldehyde, then probed overnight with primary antibodies and $4 \mathrm{~h}$ with Alexa Fluor488-labeled (Life Technologies) secondary antibodies. Images were acquired as described above.

Statistical analyses. Spearman's coefficients with associated $P$-values were calculated for correlation analyses. Two-sided one-sample or two-sample Student's $t$ tests were used to analyze the differences in miRNA and gene expression. $P<0.05$ was considered statistically significant. Data are reported as mean values \pm S.D. of at least three independent experiments.

\section{Conflict of Interest}

The authors declare no conflict of interest. 
Acknowledgements. We thank E Campi, Dr M Pennati, and Dr M Dugo for their skilled technical assistance, and $\operatorname{Dr} L$ Mariani for statistical counseling. We are grateful to the Institutional Tissue Bank and to Dr S Veneroni for sample retrieval. The study was supported by AIRC (MFAG 11542), CARIPLO Foundation, I Monzino Foundation, and an ASTIL-Proche (Regione Lombardia) grant.

1. Bartel DP. MicroRNAs: target recognition and regulatory functions. Cell 2009; 136: 215-233.

2. Gandellini $P$, Profumo V, Folini M, Zaffaroni N. MicroRNAs as new therapeutic targets and tools in cancer. Expert Opin Ther Targets 2011; 15: 265-279.

3. Gandellini P, Folini M, Longoni N, Pennati M, Binda M, Colecchia M et al. miR-205 Exerts tumor-suppressive functions in human prostate through down-regulation of protein kinase Cepsilon. Cancer Res 2009; 69: 2287-2295.

4. Gandellini P, Folini M, Zaffaroni N. Towards the definition of prostate cancer-related microRNAs: where are we now? Trends Mol Med 2009; 15: 381-390.

5. Schaefer A, Stephan C, Busch J, Yousef GM, Jung K. Diagnostic, prognostic and therapeutic implications of microRNAs in urologic tumors. Nat Rev Urol 2010; 7: 286-297.

6. Schaefer A, Jung M, Mollenkopf HJ, Wagner I, Stephan C, Jentzmik F et al. Diagnostic and prognostic implications of microRNA profiling in prostate carcinoma. Int J Cancer 2010; 126: $1166-1176$.

7. Signoretti S, Loda M. Defining cell lineages in the prostate epithelium. Cell Cycle 2006; 5 : 138-141.

8. Zhang L, Zhao W, Valdez JM, Creighton CJ, Xin L. Low-density Taqman miRNA array reveals miRNAs differentially expressed in prostatic stem cells and luminal cells. Prostate 2010; 70: 297-304.

9. Gregory PA, Bert AG, Paterson EL, Barry SC, Tsykin A, Farshid G et al. The miR-200 family and miR-205 regulate epithelial to mesenchymal transition by targeting ZEB1 and SIP1. Nat Cell Biol 2008; 10: 593-601.

10. Grisanzio C, Signoretti S. p63 in prostate biology and pathology. J Cell Biochem 2008; 103: 1354-1368.

11. Weinstein MH, Signoretti S, Loda M. Diagnostic utility of immunohistochemical staining for p63, a sensitive marker of prostatic basal cells. Mod Pathol 2002; 15: 1302-1308.

12. Ambs S, Prueitt RL, Yi M, Hudson RS, Howe TM, Petrocca F et al. Genomic profiling of microRNA and messenger RNA reveals deregulated microRNA expression in prostate cancer. Cancer Res 2008; 68: 6162-6170.

13. Bello D, Webber MM, Kleinman HK, Wartinger DD, Rhim JS. Androgen responsive adult human prostatic epithelial cell lines immortalized by human papillomavirus 18 . Carcinogenesis 1997; 18: 1215-1223.

14. Martynova E, Pozzi S, Basile V, Dolfini D, Zambelli F, Imbriano C et al. Gain-of-function p53 mutants have widespread genomic locations partially overlapping with p63. Oncotarget 2012; 3: 132-143.

15. Yu J, Peng H, Ruan Q, Fatima A, Getsios S, Lavker RM. MicroRNA-205 promotes keratinocyte migration via the lipid phosphatase SHIP2. FASEB J 2010; 24: 3950-3959.

16. Bhatnagar N, Li X, Padi SK, Zhang Q, Tang MS, Guo B. Downregulation of miR-205 and miR-31 confers resistance to chemotherapy-induced apoptosis in prostate cancer cells. Cell Death Dis 2010; 1: e105.

17. Yu X, Lin J, Zack DJ, Mendell JT, Qian J. Analysis of regulatory network topology reveals functionally distinct classes of microRNAs. Nucleic Acids Res 2008; 36: 6494-6503.

18. Vandewalle $C$, Van Roy F, Berx G. The role of the ZEB family of transcription factors in development and disease. Cell Mol Life Sci 2009; 66: 773-787.

19. Fontemaggi G, Gurtner A, Damalas A, Costanzo A, Higashi Y, Sacchi A et al. DeltaEF1 repressor controls selectively p53 family members during differentiation. Oncogene 2005; 24: $7273-7280$.
20. Patrick $B A$, Gong $X$, Jaiswal $A K$. Disruption of $\mathrm{NAD}(\mathrm{P}) \mathrm{H}$ :quinone oxidoreductase 1 gene in mice leads to 20S proteasomal degradation of p63 resulting in thinning of epithelium and chemical-induced skin cancer. Oncogene 2011; 67: 1098-1107.

21. LeBleu VS, Macdonald B, Kalluri R. Structure and function of basement membranes. Exp Biol Med 2007; 232: 1121-1129.

22. Guess CM, Quaranta V. Defining the role of laminin-332 in carcinoma. Matrix Biol 2009; 28 : 445-455.

23. Drake JM, Barnes JM, Madsen JM, Domann FE, Stipp CS, Henry MD. ZEB1 coordinately regulates laminin-332 and \{beta\}4 integrin expression altering the invasive phenotype of prostate cancer cells. J Biol Chem 2010; 285: 33940-33948.

24. Spaderna S, Schmalhofer O, Hlubek F, Berx G, Eger A, Merkel S et al. A transient, EMTlinked loss of basement membranes indicates metastasis and poor survival in colorectal cancer. Gastroenterology 2006; 131: 830-840.

25. Ke XS, Qu Y, Rostad K, Li WC, Lin B, Halvorsen OJ et al. Genome-wide profiling of histone h3 lysine 4 and lysine 27 trimethylation reveals an epigenetic signature in prostate carcinogenesis. PLoS One 2009; 4: e4687.

26. Carroll DK, Carroll JS, Leong CO, Cheng F, Brown M, Mills AA et al. p63 Regulates an adhesion programme and cell survival in epithelial cells. Nat Cell Biol 2006; 8: 551-561.

27. Härmä V, Virtanen J, Mäkelä R, Happonen A, Mpindi JP, Knuuttila M et al. A comprehensive panel of three-dimensional models for studies of prostate cancer growth, invasion and drug responses. PLoS One 2010; 5: e10431.

28. Signoretti S, Pires MM, Lindauer M, Horner JW, Grisanzio C, Dhar $S$ et al. p63 Regulates commitment to the prostate cell lineage. Proc Natl Acad Sci USA 2005; 102: $11355-11360$.

29. Koster MI, Dai D, Marinari B, Sano Y, Costanzo A, Karin M et al. p63 induces key target genes required for epidermal morphogenesis. Proc Natl Acad Sci USA 2007; 104: 3255-3260.

30. Kurata S, Okuyama T, Osada M, Watanabe T, Tomimori Y, Sato $S$ et al. p51/p63 Controls subunit alpha3 of the major epidermis integrin anchoring the stem cells to the niche. J Biol Chem 2004; 279: 50069-50077.

31. Liu A, Wei L, Gardner WA, Deng CX, Man YG. Correlated alterations in prostate basal cell layer and basement membrane. Int J Biol Sci 2009; 5: 276-285.

32. Sempere LF, Christensen M, Silahtaroglu A, Bak M, Heath CV, Schwartz G et al. Altered MicroRNA expression confined to specific epithelial cell subpopulations in breast cancer. Cancer Res 2007; 67: 11612-11620.

33. Lebanony D, Benjamin H, Gilad S, Ezagouri M, Dov A, Ashkenazi K et al. Diagnostic assay based on hsa-miR-205 expression distinguishes squamous from nonsquamous non-smallcell lung carcinoma. J Clin Oncol 2009; 27: 2030-2037.

34. Taylor RA, Toivanen R, Risbridger GP. Stem cells in prostate cancer: treating the root of the problem. Endocr Relat Cancer 2010; 17: R273-R285.

35. Lawson DA, Zong Y, Memarzadeh S, Xin L, Huang J, Witte ON. Basal epithelial stem cells are efficient targets for prostate cancer initiation. Proc Natl Acad Sci USA 2010; 107: 2610-2615.

36. Wang F, Hansen RK, Radisky D, Yoneda T, Barcellos-Hoff MH, Petersen OW et al. Phenotypic reversion or death of cancer cells by altering signaling pathways in threedimensional contexts. J Natl Cancer Inst 2002; 94: 1494-1503.

37. Testa A, Donati G, Yan $P$, Romani $F$, Huang $T H$, Viganò MA et al. Chromatin immunoprecipitation (ChIP) on chip experiments uncover a widespread distribution of NF-Y binding CCAAT sites outside of core promoters. J Biol Chem 2005; 280: 13606-13615.

38. Ortt K, Sinha S. Derivation of the consensus DNA-binding sequence for $p 63$ reveals unique requirements that are distinct from p53. FEBS Lett 2006; 580: 4544-4550.

39. Tyson DR, Inokuchi J, Tsunoda T, Lau A, Ornstein DK. Culture requirements of prostatic epithelial cell lines for acinar morphogenesis and lumen formation in vitro: role of extracellular calcium. Prostate 2007; 67: 1601-1613. 